\title{
Performance Analysis of Four Equal Port Power Splitter with Second Harmonic Suppression
}

\author{
Ankit Saxena \\ B.Tech ECE department \\ DIT, University, Dehradun \\ UK India 248001
}

\author{
Annapurna \\ B.Tech ECE department \\ DIT, University, Dehradun \\ UK India 248001
}

\author{
Brajlata Chauhan \\ AP ECE Department \\ DIT, University, Dehradun \\ UK India 248001
}

\begin{abstract}
This paper reports the performance analysis of 4 ports splitter for same frequency power output. A quarter wave $50 \Omega$ transmission lines are employed for schematic design and open circuit stubs are used for the impedance matching purpose and A passive device such as inductor may employed to reduce the $2^{\text {nd }}$ harmonic at $4.2 \mathrm{GHz}$. A high value dielectric substrate with $\varepsilon_{\mathrm{r}}$ is 9.9 used to reduce the size of transmission line at center frequency $2.1 \mathrm{GHz}$ with $500 \mathrm{MHz}$ bandwidth.
\end{abstract}

Furthermore, all the simulated scattering parameter in terms of return losses, isolation factor and coupling factor are reported here.

\section{General Terms}

Power splitter, Power combiner, Isolation loss, Return loss, coupling factor, Transmission line.

\section{Keywords}

Power divider/splitter, power combiner, ADS 2009, and simulation analysis.

\section{INTRODUCTION}

A four port power splitter is a splitter which split an input power into a four equiphase and equiamplitude outputs. When a signal enters port 5 , it spilt into equal-amplitude and equal phase output signal at port 1 , port 2 , port 3 , port 4 . With the characteristic impedance of the quarter wave lines equal to $\sqrt{2} Z_{\mathrm{o}}$, and each end of resistor has the same potential, that is zero potential difference thus no current flows through it and therefore the resistor is decoupled from input.

Furthermore, if four signals of equal amplitude and phase are applied simultaneously to the output ports of an equal split, the sum of the signals will be observed at port 5 that is the input port. Since the input signals have equal amplitude and phase, the Resistor will see the same potential at each port, and no power will be dissipated. Once the signals arrive at the junction they will constructively interfere, and the power splitter acts as a power combiner.

It also works as a combiner that is also used to combine four equal-phase and amplitude signals into a single signal in the opposite direction. Power Splitters and combiners play an important role in the design of RF and microwave circuits and their applications are various. In order to reduce $2^{\text {nd }}$ harmonic, in given proposal inductors and stubs has been used. The characteristics include the calculated reflection and isolation coefficients and insertion loss.

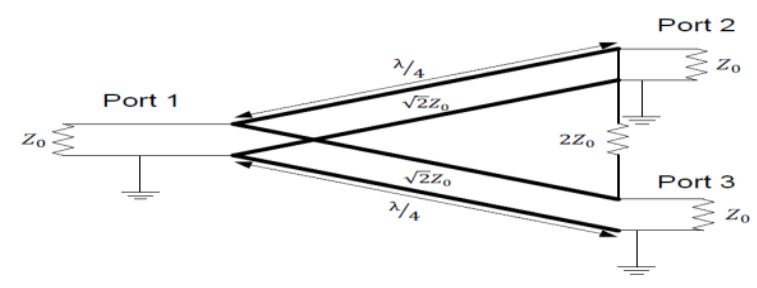

Fig 1: A power splitter with $50 \Omega$ transmission line

\section{MICROSTRIP TRANSMISSION LINE FOR POWER SPLITTER}

Transmission line provides one method of transmitting electrical energy between two points in space or antenna. With the generator connected to one end, power delivered to the matched load at the other end is given by $P l=P t e^{-2 \alpha r}$, where $\mathrm{Pt}$ is the power into the line and $\mathrm{r}$ is the distance between the two points. Transmission loss is given by

$$
10 \log \left(\frac{P t}{P l}\right)
$$

and can be minimized by analyzing the $\frac{P t}{P l}$ which is inversely proportional to $\mathrm{r}^{2}$.

The theory of transmission line is strictly applicable to only those system of conductor that have a go and return that is, those which can support a TEM wave, although hollow waveguides used in microwave region are not considered to be belonging to this category, the concept of transmission lines can be applied.

Whenever you bend a transmission line, the length of the line simply ignore the extra length that is added by the bend. If instead of circular bend, $90^{\circ}$ curved bend is used with $r>3 \mathrm{~W}$, then the effective length $h_{\mathrm{ff}}$ of the transmission line is approximately at the centerline length minus w/4.

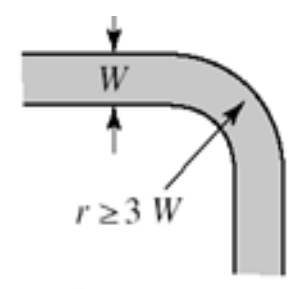

Swept bend

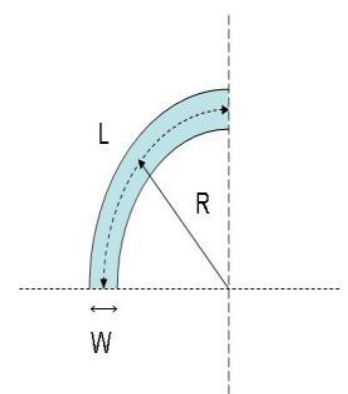

Fig 2: For effective length measurement curve bend show at $90^{\circ}$
Length calculation can be done with the help of line calculator by using ADS -Software. If the radius is greater than three times the line width, then the transmission line that is almost 
indistinguishable in impedance characteristics from a straight section.

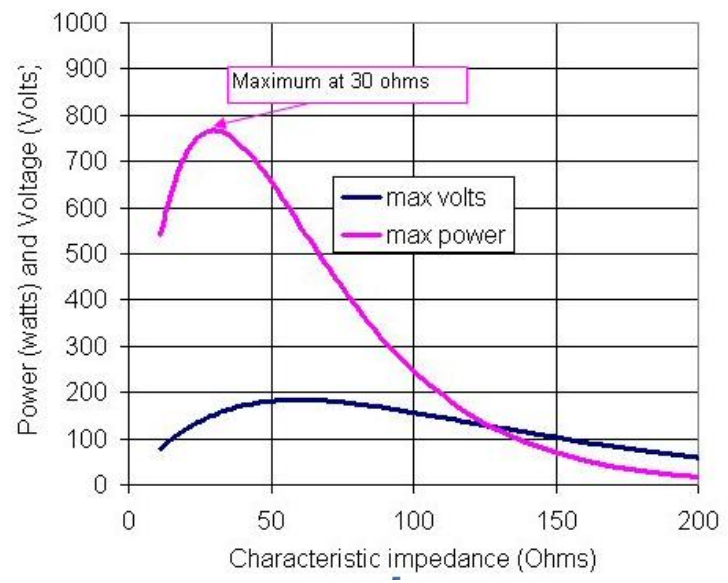

Fig 3: Characteristic impedance

Power splitters are an RF microwave device which are built in such a way so that they can provide good impedance matching at the output ports (namely port 1,2,3,4), when the input port (namely 5) is terminated with characteristic impedance of $50 \Omega$. Power splitter can be also used as comparison measurements when a prefect impedance match is achieved. Power divides the power into two antennas simultaneously.

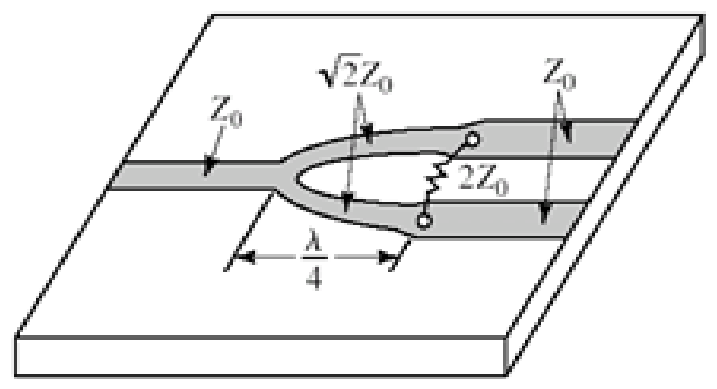

Fig 4: Characteristic of power splitter

\section{DESIGN PARAMETER}

In Advance design system (ADS) 2009 some basic parameters are used to design an RF electronic product (typically antenna).

Some parameters for four equal port power splitter are

Height $=0.76 \mathrm{~mm}$

$\mathrm{E}_{\mathrm{r}}=9.9$ Alumina $99 \%\left(\mathrm{Al}_{2} \mathrm{O}_{3}\right)$

Frequency $=2.1 \mathrm{GHz}$

Loss tangent $=0.0008$

Rough $=0$

$\mathrm{Hu}=15 \mathrm{~mm}$

Volume resistivity $=10^{14}$

Standard thickness $=0.762 \mathrm{~mm}$ for 1.524

\subsection{Line calculation for micro strip line of $\frac{\lambda}{4}$ wave transmission line \\ $\mathrm{L}=\lambda / 4 \sqrt{ }\left(\varepsilon_{\mathrm{r}}\right)$ and $\lambda=\mathrm{c} / \mathrm{f}_{0}$ \\ $\mathrm{L}=3 \times 10^{8} / 4 \times 2.10 \times 10^{9} \times \sqrt{ }(9.9) \mathrm{m}$}

$\mathrm{L}=11.35075 \mathrm{~mm}$

Total length $=22.6813 \mathrm{~mm} \quad \mathrm{~W}=24.2165 \mathrm{~mm}$

S-parameters show the relationship between input ports and output ports (or terminals) in an electrical system.

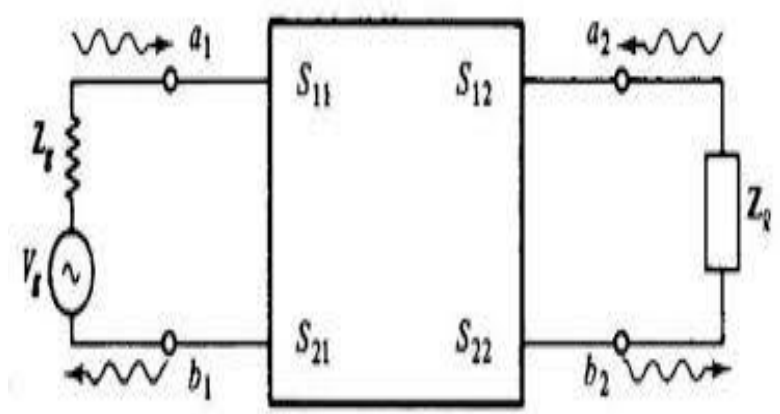

Fig 5: Two port S-Parameters

The logical variable to use at the microwave frequencies are travelling waves rather than total voltages and total currents. The parameters are expressed as

$$
\begin{aligned}
& \mathrm{B}_{1}=\mathrm{S}_{11} \mathrm{a}_{1}+\mathrm{S}_{12} \mathrm{a}_{2} \\
& \mathrm{~B}_{2}=\mathrm{S}_{21} \mathrm{a}_{1}+\mathrm{S}_{22} \mathrm{a}_{2}
\end{aligned}
$$

\section{SCHEMATIC AND LAYOUT OF THE SPLITTER}

\subsection{Schematic:}

For the four port power splitter the power is fed at port 5 (typically input port) and output port 1 , port 2 , port 3 , port 4 , and each port is terminated by the terminal with respected numbers.

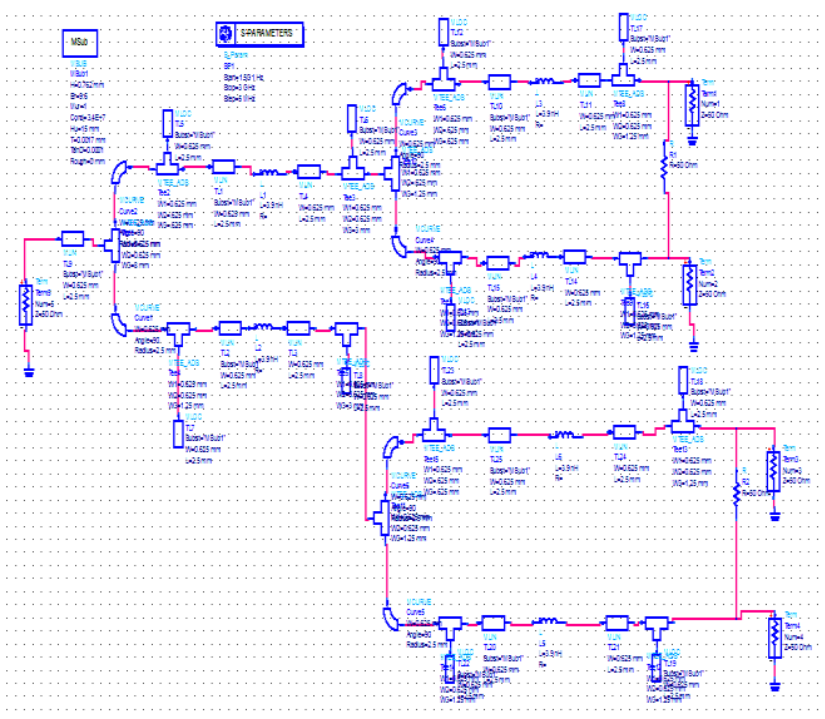

Fig 6: Schematic diagram in ADS

\subsection{Layout:}

Layout of the four ports power splitter circuit, consisting of four output ports and an input port. Blue line in the layout shows the resistance, which is an important factor for isolation loss. 


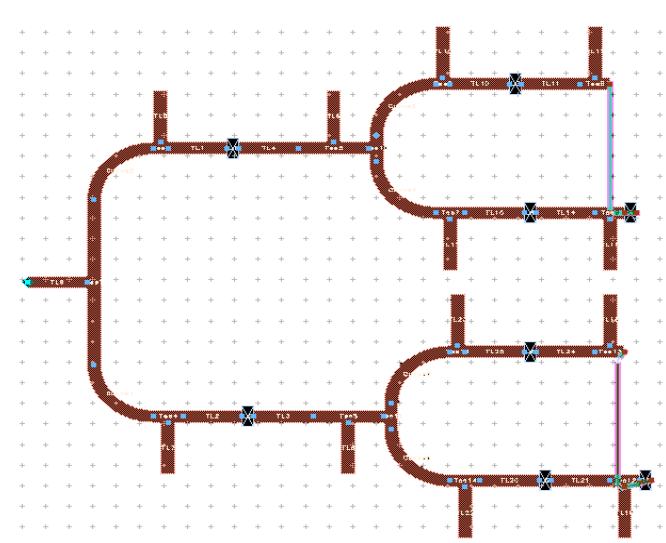

Fig 7: Layout

\subsection{Content of shown schematic diagram} Term5: Input port

Term1, Term2, Term3, Term4: Output ports

MSUB: Micro strip substrate (Alumina)

TERM: Termination $(Z=50 \Omega)$

$S$ Parameter: Scattering parameter

MCURVE: Libra micro strip curved bend

MTEE: Libra micro strip T junction

MLIN: Libra micro strip line

Lumped components-R: Resistor, I: Inductor

The S Parameter has start frequency of $1.5 \mathrm{GHz}$ with a step size of $5 \mathrm{MHz}$.

\section{SIMULATION ANALYSIS:}

Simulation analysis of four port power splitter can be explained in terms of return loss, isolation loss, coupling factor.

\subsection{Return loss $\left(\mathbf{L}_{\mathbf{r}}\right)$ :}

Return loss commonly used in conjunction with reflected signals and thus sometimes called reflection losses. If the line is lossless, the $\mathrm{L}_{\mathrm{r}}$ is the same everywhere along the line since $|\Gamma|$ is independent of position .For a lossy line $\mathrm{L}_{\mathrm{r}}$ is the function of position and varies

$\Gamma=\Gamma l e^{-2 \alpha d}$

Return loss is the measure of the reduction in the load power due to load impedance and given by

$$
\text { Return loss }=10 \log \left(\frac{1}{1-\Gamma}\right)
$$

The direct draw surface (extension .dds) window ADS is shown in given figure for return loss. Reflection coefficient

$\mathrm{S}_{11}, \mathrm{~S}_{22}, \mathrm{~S}_{33}, \mathrm{~S}_{44}, \mathrm{~S}_{55}$ and because of symmetry output ports reflection coefficient are same in numeric value.
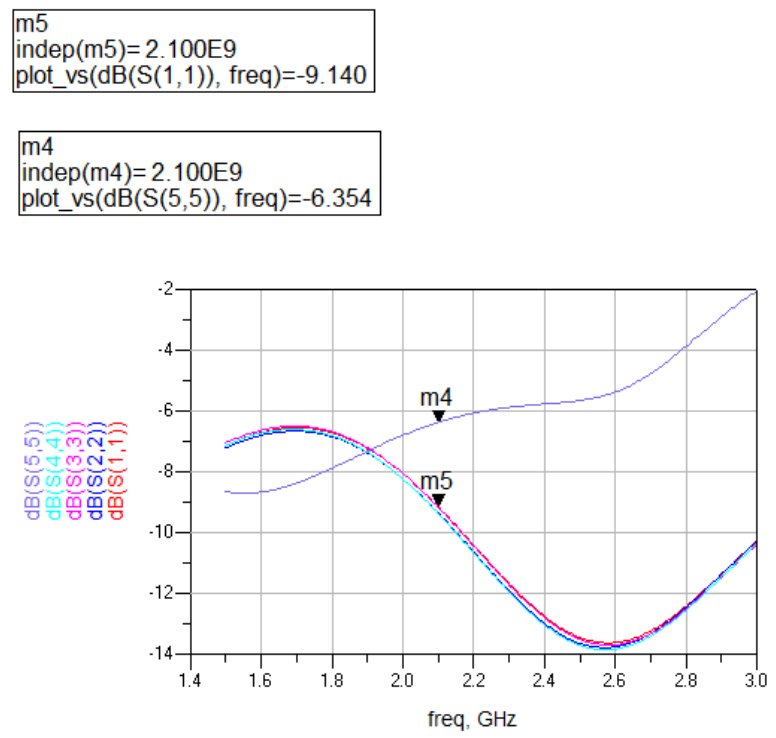

Fig 8: Return loss for input and output

\subsection{Isolation Factor:}

It is the measure of the energy loss through a transmission line as compared to direct transmission of energy without the transmission line, given by

$$
\text { isolation loss } d b=10 \log \left(\frac{E 1}{E 2}\right)
$$

Where E1 is the energy received by the load when connect directly to source without the transmission line, E2 is the energy received by the load when the transmission line is inserted between the source and load, keeping the input constant. The isolation loss is due to mismatch losses at the input and output plus the attenuation loss in the transmission line. If we assign the input at port 5 and output at ports1, 2, 3, 4 , and 5 , the isolation factor is $\mathrm{S}_{43}, \mathrm{~S}_{34}, \mathrm{~S}_{21}, \mathrm{~S}_{12}$ which are similar due to symmetry.
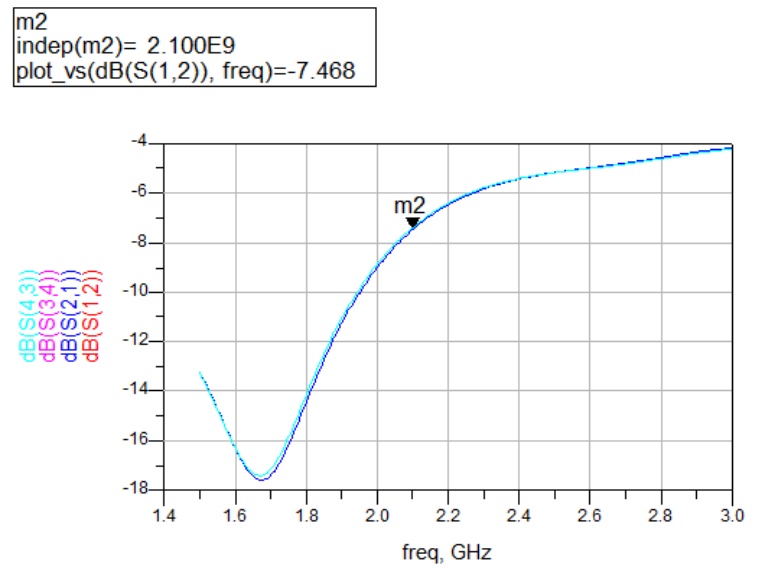

Fig 9: Isolation loss

Resistance play an important role in isolation loss, it is only due to resistance that the difference in the power can be achieved.

\subsection{Coupling factor:}

Coupling factor is the measure of how much of the incident power is being sampled.it is also defined as the how much power is coupled between the input and the output ports. 
Corresponding to previous port assigning coupling factors are $\mathrm{S}_{15}, \mathrm{~S}_{25}, \mathrm{~S}_{35}, \mathrm{~S}_{45}$ and due to symmetry have same value.
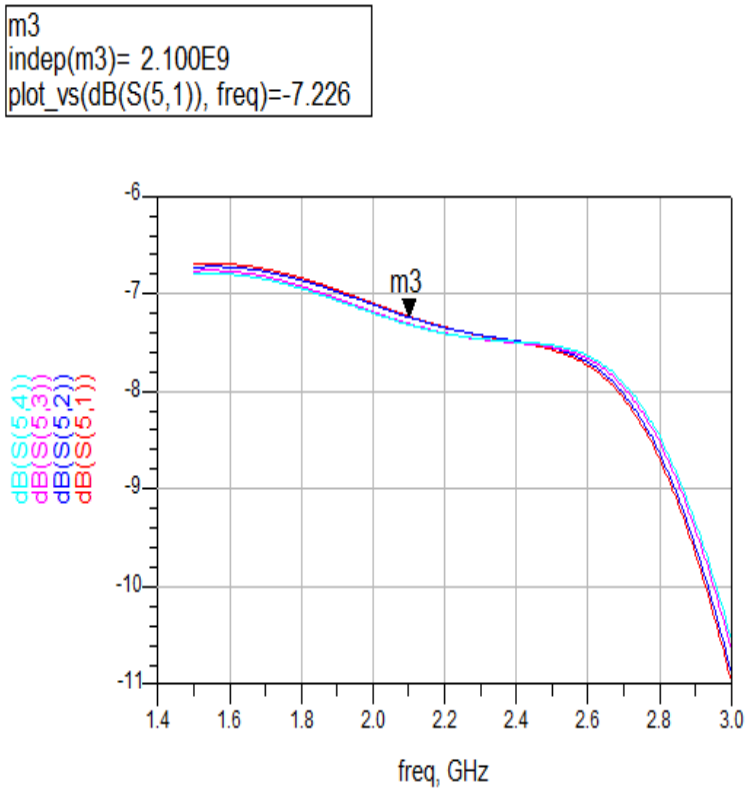

Fig 10: Coupling factor

\section{RESULT}

Layout can be automatically generate in advance design system (ADS) in layout window for the achieved schematic diagram.

The simulated parameters of four port power splitter at frequency $2.1 \mathrm{GHz}$ are as: Isolation factor is minus $7.46 \mathrm{db}$, return loss for input port is minus $6.35 \mathrm{db}$ and for output port is minus $9.14 \mathrm{db}$, coupling factor is minus $7.22 \mathrm{db}$.

\section{CONCLUSION}

This paper reported performance analysis of four equal port power splitter with second harmonic suppression along with its return loss, isolation loss and coupling factor through a simulated analysis in ADS.

Furthermore this design may be used for power combining and splitting application for quadrature multiplexing, transmitter, receiver and antenna power combining application at center frequency $2.1 \mathrm{GHz}$.

\section{REFERENCES}

[1] F. Noriega and P. J. González, "Designing LC Wilkinson power splitters,"RFdesign.com Aug. 2002

[2] J. Li, "Novel Design of Wilkinson Power Splitters With Arbitrary Power Division Ratios," IEEE Transactions on Industrial Electronics, Vol. 58 Issue 6, pp. 2541-2546, 2011

[3] G. S. Makineni and W. T. Joines, "Comparison of broadband performance of two-way power splitters and combiners," Microw. Opt.Technol. Lett., vol. 17, no. 1, pp. 29-37, Jan. 1998.

[4] GPP TS 25.101 V5.2.0 (2002-03) Technical Specification Group Radio Access Networks; UE Radio Transmission and Reception (FDD) (C) 2002.

[5] T.G. Bryant and J.A.Weiss, "Parameters of Microstrip Transmission Lines and Coupled Pairs of Microstrip Lines," Vol. 16 Issue 12, pp. 1021-1027, 1988

[6] Microwave Power Splitter and Couplers Tutorial, Marki Microwave, Inc. Morgan Hill, CA.

[7] L. Wu, Z. Sun, H. Yilmaz, and M. Berroth, "A Dual-Frequency Wilkinson Power Splitter," IEEE Transactions on Microwave Theory and Techniques, Vol. 54 Issue 1, pp. 278-284, 2006

[8] E.J. Wilkinson, “An N-Way Hvbrid Power Splitter, " IRE Trans. on Microwave Theory and Techniques, VO1. MTT-8, pp. 116-118 (January 1960).Progress In Electromagnetics Research

[9] ] E. J. Wilkinson, "An N-Way Hybrid Power Splitter," IRE Transactions on Microwave Theory and Techniques, Vol. 8 Issue 1, pp. 116-118, 1960.

[10] A. Wentzel, V. Subramanian, A. Sayed, and G. Boeck, "Novel Broadband Wilkinson Power Combiner," Proceedings of the 36th European Microwave Conference, pp. 212215, 2006

[11] Chen, H. and Y. X. Zhang, \A novel compact planar six-way power splitter using folded and hybridexpanded coupled lines,"Progress In Electromagnetics Research, Vol. 76, 243\{252, 2007.

[12] Y. Konishi, Microwave Electronic Circuit Technology, New York, New York: Marcel Dekker Inc. $\quad$ 273-292, 1998. 